\title{
Um Estudo Exploratório dos Fatores de Adoção dos Ecossistemas de Software em Nuvem
}

\author{
Emanuel A. Rodrigues, Antonilson S. Alcantara, Filipe D. Coelho, \\ Cleidson R. B. de Souza \\ Universidade Federal do Pará (UFPA) - Belém, PA - Brasil \\ emanorodrigues@gmail.com, antonilsonalcantaralgmail.com, \\ filipe.bcdc@gmail.com, cleidson.desouzalacm.org.
}

\begin{abstract}
Resumo. Nos últimos anos, um número crescente de empresas de software tem adotado estratégias para atrair desenvolvedores externos para suas plataformas. Essas empresas tornam suas plataformas mais flexíveis e atraentes, de modo a proporcionar o surgimento dos chamados ecossistemas de software. O estabelecimento destes ecossistemas já ocorreu no mercado de dispositivos móveis e agora está ocorrendo no mercado de computação em nuvem. Este artigo tem o objetivo de entender os critérios utilizados pelos desenvolvedores de aplicações para nuvem a adotar uma determinada plataforma e o ecossistema associado. Isto é feito através da identificação de diferentes fatores que influenciam os desenvolvedores a escolher uma determinada plataforma em nuvem. Estes fatores foram identificados a partir de um estudo qualitativo baseado em 16 entrevistas semiestruturadas com desenvolvedores em nuvem. A análise dos dados revelou que fatores técnicos, sociais, individuais e de negócios são importantes para a adoção ou rejeição de uma determinada plataforma pelos desenvolvedores de software.
\end{abstract}

\section{Introdução}

Segundo Slawik (2018), nos últimos anos, o paradigma da computação em nuvem tornouse presente em diversos segmentos, incluindo a Tecnologia da Informação. Isto possibilitou a transformação na maneira como os recursos computacionais, redes e armazenamento de dados são oferecidos. Esta transformação da computação física para a nuvem introduziu novos conceitos, como por exemplo, a implementação de aplicativos imutáveis, microserviços em containers, frameworks ubíquos baseados em Java Script, entre outros.

As plataformas em nuvem têm se tornado mais flexíveis, proporcionando o surgimento dos chamados ecossistemas de software (BOSCH, 2009). No contexto de ecossistemas de software as empresas são incentivadas a abrir suas plataformas com o objetivo de atrair desenvolvedores externos e, assim, obterem novas contribuições para seus produtos e serviços. Isto contrasta com o modelo anterior onde softwares eram desenvolvidos por um grupo de engenheiros que trabalhavam de maneira isolada em suas organizações e sem a influência e/ou participação do mundo externo.

O conceito de ecossistema de software ganhou força especialmente depois das plataformas iOS da Apple e Android da Google para dispositivos móveis. Mais recentemente, os ecossistemas de software em nuvem começaram a surgir devido ao 
mercado existente nesta categoria. Assim, exemplos de ecossistemas para a nuvem incluem Amazon AWS, Microsoft Azure, IBM Bluemix e Google Cloud Platform (GCP), cada um destes com centenas de aplicações implementada por desenvolvedores externos.

Quando as organizações deixam de desenvolver seus produtos e serviços de forma isolada e passam a fechar parcerias com outras organizações ou desenvolvedores independentes, elas alcançam um grande nível de evolução tecnológica (BERK et al., 2010). Como exemplo, pode-se citar o Amazon AWS, que atualmente oferece em torno de setenta serviços em nuvem para mais de um milhão de clientes corporativos. Além disso, a Amazon já atraiu mais de onze mil membros em sua rede de parceiros, o que gerou uma receita de 7,7 bilhões de dólares em 2019 (COMPUTERWORLD, 2019).

Tendo em vista as tendências de inovações envolvendo ecossistemas de software em nuvem, este trabalho apresenta uma pesquisa que pretende contribuir no entendimento do processo de adoção de um determinado ecossistema ao identificar os fatores que influenciam essa escolha incluindo fatores técnicos, sociais, individuais e de negócios. Isto é feito através de um estudo qualitativo baseado em 16 entrevistas semiestruturadas com desenvolvedores de software para a nuvem.

O restante deste artigo está organizado da seguinte forma. Na próxima seção, os principais conceitos sobre Ecossistemas de Software são descritos e um breve resumo dos principais conceitos da computação na nuvem são apresentados. A seção 3 apresenta a metodologia utilizada para a coleta e análise dos dados enquanto as próximas seções apresentam os resultados obtidos e a discussão, respectivamente. Finalmente, a última seção aponta direcionamentos para trabalhos futuros.

\section{Conceitos Básicos}

\subsection{Ecossistemas de software}

A partir de uma analogia com o conceito de ecossistema da área de ecologia, Bosch (2009) define ecossistemas de software como um conjunto de soluções de software e uma comunidade de desenvolvedores independentes que trabalham de maneira cooperativa para entregar serviços à comunidade de usuários, compondo serviços relevantes para suas necessidades. De maneira similar, Jansen et al. (2009) define um ecossistema de software como "um conjunto de atores que agem como uma unidade e se inter-relacionam com um mercado compartilhado de software e serviços, juntamente com as relações entre eles". Nesse sentido, quando as organizações cessam o desenvolvimento de seus produtos e serviços isoladas e associam-se com outras organizações ou desenvolvedores independentes, elas alcançam um alto nível de evolução tecnológica (BERK et al., 2010).

De acordo com Jansen e Cusumano (2012), existem três papéis principais em um ecossistema: (i) Keystone é a empresa que possui o ecossistema de software. Por exemplo, no ecossistema do Azure, a keystone é a Microsoft que fornece sua plataforma e todos os serviços associados a ela; (ii) Niche players são as empresas que trabalham nas plataformas fornecidas pelas keystones, criando ou aprimorando seus produtos e serviços; e, finalmente, os (iii) End users que, como o nome sugere, são os consumidores dos produtos e serviços fornecidos pelo ecossistema de software. Em particular, este trabalho foca nos desenvolvedores de software que atuam como niche players, visto que o interesse do mesmo é entender os motivos que levam tais desenvolvedores a adotar, ou não, um determinado ecossistema de nuvem. 
Barbosa et al.(2013) afirmam que um ecossistema de software deve ser entendido em três dimensões: a) técnica, que se relaciona com a plataforma de software e sua infraestrutura; b) negócio, que se relaciona com seu modelo de negócio, estratégia de licenciamento e de parceiros; e c) social, que é como os atores interagem entre si nos ecossistemas, isto é, a comunidade em volta do ecossistema. Entretanto, é importante ressaltar que tais dimensões estão inter-relacionadas, ou seja, elas se afetam mutuamente. Conforme será discutido na seção 3 , esta visão de dimensões foi utilizada neste trabalho.

\subsection{Computação em Nuvem}

Os anos 2000 ficaram marcados pelo início da era "ponto com". A partir de então, a disponibilidade de múltiplas arquiteturas, largura de banda de alta velocidade e padrões de interoperabilidade criaram um ambiente ideal para a proliferação da computação em nuvem (SLAWIK, 2018). Ressalta-se que a realização de uma rede global de alta velocidade e um modelo de negócios baseado em serviços públicos são os dois principais princípios por trás da computação em nuvem (GONZALEZ; KRISHNAN, 2015).

Para Gonzalez e Krishnan (2015), a computação em nuvem consiste em abstrair a infraestrutura e outros recursos associados e oferecê-los como serviços, normalmente com pagamento sob demanda. O serviço pode ser consumido por humanos ou por outros sistemas de software (via APIs) e os usuários precisam apenas de um navegador web para acessar tais serviços. Tal abstração de infraestrutura é geralmente realizada por meio de um processo técnico chamado virtualização.

A computação em nuvem difere da computação tradicional em diversos aspectos como infraestrutura, manutenção e mão de obra (GONZALEZ; KRISHNAN, 2015). Na computação em nuvem, as empresas usuárias não se preocupam com recursos de infraestrutura como CPU, RAM, servidores ou data centers, uma vez que esses recursos estão na nuvem e são adquiridos conforme demanda. Em relação à manutenção, a computação tradicional deve ter assistência aos recursos tanto de hardware quanto de software. Já na nuvem, a manutenção é praticamente zero, pois os recursos estão em uma nuvem pública. Observa-se ainda que, na computação tradicional, as empresas têm de contratar especialistas em banco de dados, devops, redes etc, para entregar um serviço eficiente a seus clientes, enquanto que na computação na nuvem, os recursos financeiros que iriam para a mão de obra podem ser economizados e distribuídos para outros benefícios, potencialmente aumentando o ROI sobre os produtos e serviços oferecidos.

\section{Metodologia}

O método científico desta pesquisa foi o exploratório, do tipo levantamento de dados, com abordagem qualitativa, visto que se baseia em interações com pessoas que conduzem uma determinada atividade. Para alcançar o objetivo proposto - o entendimento dos fatores que influenciam na adoção de ecossistemas em nuvem - um estudo qualitativo por meio de entrevistas semiestruturadas foi realizado. Especificamente, entrevistas semiestruturadas foram conduzidas com 16 desenvolvedores (treze homens e três mulheres) que desenvolvem para as plataformas de nuvem AWS (8), Azure (5) e GCP (3). Desse total, doze eram profissionais com mais de três anos de experiência no desenvolvimento para nuvem e o restante com menos de dois anos de experiência. Os entrevistados tinham entre 21 e 35 anos de idade, sendo que um trabalha nos EUA e o restante no Brasil. As entrevistas foram realizadas no período de fevereiro a maio de 2019, 
gravadas com permissão dos entrevistados e tiveram a duração de 18 a 32 minutos. A Tabela 1 abaixo apresenta as características dos participantes deste estudo. Por questões de confidencialidade, seus nomes não são revelados.

\begin{tabular}{|c|c|c|c|c|c|}
\hline Participantes & Idade & Sexo & Formação & $\begin{array}{l}\text { T. de Exp. } \\
\text { na nuvem }\end{array}$ & $\operatorname{Ecossistema(s)}$ \\
\hline Pessoa $1[\mathrm{P} 1]$ & 35 & $\mathrm{H}$ & $\begin{array}{l}\text { Pós-graduação em } \\
\text { Análise e } \\
\text { Desenvolvimento de } \\
\text { Sistemas }\end{array}$ & 6 anos & Microsoft Azure \\
\hline Pessoa 2 [P2] & 37 & $\mathrm{H}$ & $\begin{array}{l}\text { Mestrado em Ciência } \\
\text { da Computação }\end{array}$ & 3 anos & $\begin{array}{l}\text { Microsoft Azure, } \\
\text { Amazon AWS e } \\
\text { GCP - Google } \\
\text { Cloud Platform }\end{array}$ \\
\hline Pessoa 3 [P3] & 36 & $\mathrm{H}$ & $\begin{array}{l}\text { Doutorado em } \\
\text { Ciência da } \\
\text { Computação }\end{array}$ & 5 anos & $\begin{array}{l}\text { Amazon AWS, } \\
\text { GCP - Google } \\
\text { Cloud Platform e } \\
\text { Heroku }\end{array}$ \\
\hline Pessoa 4 [P4] & 24 & $\mathrm{H}$ & $\begin{array}{l}\text { Pós-graduação em } \\
\text { Análise de Sistemas }\end{array}$ & 2 anos & IBM Cloud \\
\hline Pessoa 5 [P5] & 39 & $\mathrm{H}$ & $\begin{array}{l}\text { Doutorado em } \\
\text { Ciência da } \\
\text { Computação } \\
\end{array}$ & 3 anos & Amazon AWS \\
\hline Pessoa 6 [P6] & 26 & M & $\begin{array}{l}\text { Pós-Graduação em } \\
\text { Desenvolvimento de } \\
\text { Sistemas Web }\end{array}$ & 1 ano & Amazon AWS \\
\hline Pessoa 7 [P7] & 23 & $\mathrm{H}$ & Graduando em Dev. & 1 ano & Amazon AWS \\
\hline Pessoa 8 [P8] & 22 & & Graduando em Dev. & 1,5 anos & $\begin{array}{l}\text { Microsoft Azure } \\
\text { e Amazon AWS }\end{array}$ \\
\hline Pessoa 9 [P9] & 29 & M & Graduando em Dev. & 2,5 anos & Amazon AWS \\
\hline $\begin{array}{l}\text { Pessoa } 10 \\
{[\mathrm{P} 10]}\end{array}$ & 24 & $\mathrm{H}$ & Graduando em Dev. & 2 anos & Amazon AWS \\
\hline $\begin{array}{l}\text { Pessoa } 11 \\
{[\mathrm{P} 11]}\end{array}$ & 28 & $\mathrm{H}$ & $\begin{array}{l}\text { Grad. em Engenharia } \\
\text { da Computação }\end{array}$ & 8 meses & Amazon AWS \\
\hline $\begin{array}{l}\text { Pessoa } 12 \\
{[\mathrm{P} 12]}\end{array}$ & 25 & M & Grad. em SI & 6 meses & $\begin{array}{l}\text { Amazon AWS e } \\
\text { Microsoft Azure }\end{array}$ \\
\hline $\begin{array}{l}\text { Pessoa } 13 \\
{[\mathrm{P} 13]}\end{array}$ & 21 & $\mathrm{H}$ & $\begin{array}{l}\text { Grad. em Engenharia } \\
\text { da Computação }\end{array}$ & 2 anos & Amazon AWS \\
\hline $\begin{array}{l}\text { Pessoa } 14 \\
{[\mathrm{P} 14]}\end{array}$ & 28 & $\mathrm{H}$ & $\begin{array}{l}\text { Mestre em } \\
\text { Engenharia da } \\
\text { Computação }\end{array}$ & 1 ano & $\begin{array}{l}\text { GCP - Google } \\
\text { Cloud Platform }\end{array}$ \\
\hline $\begin{array}{l}\text { Pessoa } 15 \\
{[\mathrm{P} 15]}\end{array}$ & 28 & $\mathrm{H}$ & $\begin{array}{l}\text { Pós-graduado em } \\
\text { Desenvolvimento de } \\
\text { software }\end{array}$ & 3 anos & Microsoft Azure \\
\hline
\end{tabular}




\begin{tabular}{|l|c|c|l|c|l|}
\hline $\begin{array}{l}\text { Pessoa 16 } \\
{[\mathrm{P} 16]}\end{array}$ & 26 & $\mathrm{H}$ & Graduando em Dev. & 6 meses & $\begin{array}{l}\text { GCP - Google } \\
\text { Cloud Platform }\end{array}$ \\
\hline
\end{tabular}

\section{Tabela 1. Características dos participantes entrevistados}

O objetivo das entrevistas era identificar os pontos positivos e negativos que influenciam os desenvolvedores na adoção de um determinado ecossistema em nuvem. Os pontos positivos são aspectos que os desenvolvedores relataram como sendo benéficos para adoção e permanência em um determinado ecossistema. Por exemplo, "Achei muito legal o ambiente de desenvolvimento da Azure, como eu já desenvolvia no Visual Studio, consegui sem problemas migrar para Azure que é praticamente a mesma coisa que o Visual". Neste caso, o ambiente de desenvolvimento é um aspecto técnico que influencia na adoção da plataforma Azure. Por outro lado, os aspectos negativos são aqueles que de alguma forma dificultam tanto a adoção quanto a permanência em um ecossistema. Por exemplo, "O que me fez deixar a IBM Cloud, foi a comunidade, sempre que tinha dúvida recorria para ela sem êxito, e via meus amigos dizendo que a comunidade da Google $G C P$, era bem engajada". Neste exemplo, a comunidade de desenvolvedores foi um fator que afetou de maneira negativa a permanência do(a) informante na IBM Cloud.

As entrevistas semiestruturadas foram transcritas e analisadas utilizando as técnicas de codificação (aberta e axial) da Teoria Fundamentada em Dados (Strauss; Corbin, 2008). Em outras palavras, a partir da leitura de cada trecho das entrevistas, o referido trecho foi classificado em um dos quatros aspectos a seguir: social, negócio, técnico e individual. Esta classificação se baseia no trabalho de Barbosa et al. (2013) discutido na subseção 2.1 que descreve três dimensões iniciais: a técnica, a de negócio e a social. Entretanto, é importante considerar o aspecto individual dos desenvolvedores de software visto que o foco deste trabalho é na motivação dos mesmos para adotar, ou permanecer, em um determinado ecossistema. Além da classificação nestes quatro aspectos, cada trecho de entrevista foi classificado indicando se o mesmo se referia a pontos positivos ou negativos de um determinado ecossistema em nuvem. Em resumo, a análise qualitativa nos permitiu compreender quais fatores influenciam os desenvolvedores a escolher um ecossistema de software em nuvem.

\section{Resultados}

Nesta seção são apresentados os resultados desta pesquisa separando-os em quatros aspectos: social, negócio, técnico e individual, bem como indicando os pontos positivos e negativos dos mesmos. É importante ressaltar que os diversos fatores foram divididos nestes quatro aspectos visando o entendimento do problema, mas na prática os entrevistados foram influenciados por diversos fatores ao mesmo tempo. Além disso, os resultados deixaram claros que alguns fatores são positivos para um entrevistado, mas negativos para outros. Neste caso, os aspectos foram classificados como ambíguos.

\subsection{Aspectos de Negócio}

A seguir são ilustrados exemplos de como os aspectos de negócio influenciam de forma positiva na decisão de iniciar o desenvolvimento para um dado ecossistema em nuvem:

"Na verdade, não tive escolha. Existe um acordo entre a Microsoft e a empresa onde eu trabalho. Então, usamos o Azure por um custo muito barato (quase de graça)". [P3] 
O informante P3 sugere a existência de uma parceria entre grandes empresas e os keystones que deixam a precificação das plataformas atraente. Esta é uma estratégia usada pelas keystones, que como pode ser visto, traz resultados positivos para a mesma. Enquanto isto, P8 mencionou:

"Eu poderia dizer que fui influenciado pelo mercado e também foi a decisão da empresa que eu trabalhei na época. Além disso, foi a minha primeira opção do mercado há 3 anos e acredito que há muitas razões para isso: Boa documentação, tem uma enorme comunidade online, o preço (quando bem utilizado) é justo”. [P8]

Os comentários dos desenvolvedores P3 e P8 mostram como fatores ambientais da empresa, como política organizacional, modelo de negócios e normativas podem levar o desenvolvedor a aderir a um determinado ecossistema. É importante ressaltar que o outro aspecto (a comunidade online) mencionado pelo informante P8 será detalhado mais adiante quando os aspectos sociais forem discutidos.

A precificação, mudança de mercado, futuras oportunidades de emprego (mercado de trabalho) no ecossistema também são aspectos de negócio que podem influenciar de maneira positiva e negativa, como os excertos abaixo ilustram:

"Estamos mesclando entre as plataformas, por conta das cotas, como não temos dinheiro não temos como investir no projeto, então por exemplo minha cota da Google acabou e como eu estava estudando AWS, passei a subir tudo pela AWS, mas meu colega disponibilizou um ambiente para produção que seria para apresentação, mostrar o case, agora o desenvolvimento é em AWS”. [P2]

"Certo dia eu peguei meu celular as cinco da manhã e tinha uma mensagem você gastou tantos dólares, eu falei 'eita', sai correndo liguei o notebook e desliguei, então falei para o pessoal: 'gente, ambiente de DEV agora só mês que vem' e foi interessante por causa disso.” [P6]

“E os aspectos de negócio, foram importantes nessa parte, tipo na Google ... eles te dão uma grana, acho que mil reais, para você ir gastando conforme o tempo, no caso o meu gasto, eu subi a máquina e esqueci de desligar e acabou, foi basicamente isso, na AWS foi um pouco diferente, eu consegui criar um alarme, porque é assim, são doze meses, mas assim 750 horas de funcionamento das máquinas e ai eu criei um alarme que quando chegasse $70 \%$ ele avisa para eu poder desligar, tipo a gente só liga as máquinas em horários específicos, esse mês não vamos usar o ambiente dev porque o orçamento acabou”. [P16]

"O que influenciou a entrar no mundo da cloud, foi basicamente a mudança de mercado, andei percebendo pelo linkedin e outras redes socais de emprego, que as vagas estavam mudando, tinha muita vaga em aberto para desenvolvedores em cloud”. [P12]

"Outro fator interessante é que ele AWS disponibiliza tipo uma calculadora, você coloca o tipo de máquina que você quer, algumas informações complementares e aí ele te dá uma estimativa de gastos por hora, por mês, eu tenho pra mim, que essa ferramenta facilita em vários projetos e eles sempre deixam expostos as calculadoras deles, ela é bem intuitiva”. [P6] 
A fala do informante [P2] revela que a precificação é relevante, pois os desenvolvedores independentes não dispõem de tantos recursos financeiros para sustentar seus projetos, fazendo que eles recorram a múltiplas plataformas em busca do que tem de melhor e mais barato entre elas. Por outro lado, percebe-se que a precificação pode ser benéfica para o desenvolvedor, uma vez que é importante que as plataformas sejam especificas quanto a forma de cobrança, locação dos serviços e tempo disponibilizado, viabilizando o controle direto por meio do usuário.

É possível perceber também, conforme a fala do informante [P6], que a "calculadora" de custos dos serviços e recursos das plataformas é importante, pois permite que os desenvolvedores gerenciem seus gastos. Por outro lado, alguns desenvolvedores não precisam controlar os custos de alguns serviços da plataforma, uma vez que existe um setor responsável em suas organizações para lidar com isto. Ou seja, existe uma gestão na empresa que lida com os preços das plataformas.

"Não tenho noção dos valores praticados pelas plataformas, sou apenas desenvolvedor e não tenho conhecimento dessa parte, na minha empresa tem um setor financeiro responsável por isso." [P8]

O entrevistado [P12] ressalta a importância do mercado de trabalho com o surgimento de vagas na área de desenvolvimento em nuvem, o que fez com que ele buscasse aprendizado sobre o tema.

A Tabela 2 a seguir resume os diferentes aspectos de negócios e se eles influenciam de maneira positiva, negativa ou ambígua na adoção de um ecossistema em nuvem.

\begin{tabular}{|l|l|l|}
\hline Positivo & Negativo & Ambíguos \\
\hline Evangelização [P16] & & Precificação [P8, P6] \\
\hline Mercado de trabalho [P12] & & Rentabilidade [P8] \\
\hline Gestão da empresa [P3] & & \\
\hline
\end{tabular}

Tabela 2. Principais aspectos de negócio.

\subsection{Aspectos Técnicos}

Os desenvolvedores entrevistados nos indicaram que a adoção de uma plataforma em nuvem também é influenciada por questões técnicas das mesmas. Os trechos a seguir mostram como o desenvolvedor P1 foi influenciado positivamente pela API disponível, a infraestrutura do ecossistema e migração para outras plataformas. Enquanto isto, P4 afirma que a ideia da IDE estar disponível na nuvem deixando o código mais seguro e disponível quando for solicitado é um aspecto positivo para ele.

"O principal aspecto que me levou à AWS é o aspecto técnico: API poderosa, muitas ferramentas, grande oportunidade de automatizar a infraestrutura.”. [P1]

“Achei muito legal a ideia de não perder meus códigos por alguma pane na minha máquina e gostei”. [P4]

Os trechos a seguir mostram que a infraestrutura, documentação, ferramentas e serviços oferecidos pelas plataformas podem ser um aspecto que influencia tanto de maneira positiva quanto de maneira negativa um desenvolvedor a aderir para a plataforma: 
"A infraestrutura da Google fez que eu mudasse de plataforma, não sei te dizer porque, mas não me acostumei com os serviços dela, daí então, fui para a Amazon e aprendi a usar as ferramentas bem rápido, parece que a Google não sabe organizar o ambiente de trabalho do desenvolvedor, pode ser isso. Os ambientes que eu posso dizer que são bastante intuitivos, são da AWS que eu uso bastante e também da Azure, mas este eu uso de vez em quando”. [P11]

"Bom, já testei algumas plataformas e sem dúvida a Amazon AWS tem a melhor documentação, tudo é muito bem explicado e dividido, você pode ir acompanhando que vai aprender bastante, é tipo um passo a passo do desenvolvimento para cloud, eles explicam em detalhes como utilizar as ferramentas disponíveis, os desenhos são bem ilustrados e cheio de exemplos". [P6]

"Tive dificuldade com a documentação da Azure, talvez seja por causa do meu inglês que não é muito bom, não consegui na época material em português." [P13]

“Achei muito legal o ambiente de desenvolvimento da Azure, como eu já desenvolvia no Visual Studio, consegui sem problemas migrar para Azure que é praticamente a mesma coisa que o Visual”. [P14]

"O desenvolvimento na nuvem é diferente do desenvolvimento tradicional, antes eu ficava tendo que configurar servidor e tudo mais, agora eu desenvolvo um aplicativo, um serviço sem me preocupar com servidor, a plataforma disponibiliza tudo para o desenvolvedor, sem conta dos inúmeros serviços que a plataforma oferece, tipo máquina virtual, inteligência artificial e etc”.'[P6]

A Tabela 3 resume como os diferentes aspectos técnicos influenciam na adoção de um ecossistema na nuvem.

\begin{tabular}{|l|l|l|}
\hline Positivo & Negativo & Ambíguos \\
\hline Boas APIs (P4, P1) & & Documentação (P6, P13) \\
\hline $\begin{array}{l}\text { Programação sem Servidor } \\
\text { (P1, P14) }\end{array}$ & $\begin{array}{l}\text { Infraestrutura de } \\
\text { Desenvolvimento (P1, P11) }\end{array}$ \\
\hline $\begin{array}{l}\text { Ambiente de Desenvolvimento } \\
\text { (P14, P1) }\end{array}$ & & \\
\hline
\end{tabular}

Tabela 3. Principais aspectos técnicos.

\subsection{Aspectos Sociais}

Os trechos a seguir mostram como aspectos sociais influenciam na adoção das plataformas em nuvem. A mudança de mercado fez com que muitos desenvolvedores web migrassem para a nuvem em buscas de novas oportunidades no mercado e alavancagem na carreira. A comunidade teve um papel essencial, tanto positivo quanto negativo, como os excertos das entrevistas com P5, P8 e P15 mostram a seguir:

"Eu posso dizer que a comunidade foi de grande importância para que eu começasse a desenvolver para a Amazon AWS, por que ela diminuiu muito minha curva de aprendizado, em pouco tempo eu já estava criando meu primeiro micro serviço". [P8] 
"O que me fez deixar a IBM Cloud, foi a comunidade, sempre que tinha dúvida recorria para ela sem êxito, e via meus amigos dizendo que a comunidade da Google - GCP, era bem engajada, que eles faziam encontros semanais na minha cidade, daí foi então que resolvi deixar a IBM e ir para a Google, é sem dúvida enriqueci meus conhecimentos nessa mudança, agora participo sempre das meetup que a comunidade organiza e aprendo bastante”. [P15]

"Meu grupo de amigos é difundida por causa das empresas, cada um trabalha em uma empresa, cada um tem alguma coisa para mostrar, então você vê que seus colegas trazem alguma experiência, cada um de uma plataforma; e a AWS é a que eu mais ouvir falar e a que mais me interessou, tipo você está com esta plataforma e está sendo uma experiência boa, bom então se é bom, vamos ver se vai funcionar para mim também, e foi por esse motivo". [P5]

"Sempre que possível estou, tanto postando dúvidas e também tirando dúvidas; é um troca grande de experiência que enriquece seu conhecimento sobre a plataforma. "[P12]

"O GLP da Google está chamando atenção pela sua evangelização, assim como, Open Shift, o Microsoft Azure não achei atrativo a um ano atrás.”[P3]

Como pode ser observado, as comunidades online e locais são de grande importância para que os desenvolvedores consigam avançar no desenvolvimento de aplicativos na nuvem. Percebe-se também um movimento de meetups, isto é, encontros entre desenvolvedores e interessados que buscam a troca de conhecimento e aprendizado entre os participantes. Estes eventos estão sendo disseminados pelo Brasil e pelo mundo.

Conforme trecho do [P12], percebe-se que a comunidade serve como grande troca de experiências entre os desenvolvedores individuais e de empresas, esse feedback faz com que aumente a curva de aprendizado sobre a plataforma em questão.

\begin{tabular}{|l|l|l|}
\hline Positivo & Negativo & Ambíguos \\
\hline $\begin{array}{l}\text { Evangelização da } \\
\text { plataforma (P3, P5) }\end{array}$ & & $\begin{array}{l}\text { Engajamento da } \\
\text { comunidade (P15, P12, P8, } \\
\text { P5) }\end{array}$ \\
\hline
\end{tabular}

Tabela 4. Principais aspectos sociais.

\subsection{Aspectos Individuais}

Por fim, são apresentados aspectos individuais, onde os entrevistados relataram que estão desenvolvendo para nuvem porque gostam de aprender coisas novas e desafiadoras:

"Já desenvolvo a um bom tempo, antes mesmo de entrar na faculdade, e resolvi por questões próprias ir para a cloud, achei um desafio interessante e resolvi arriscar". [P13]

"Comecei no mundo da cloud por desafios, queria saber como funcionava, até então desenvolvia para web e achei interessante desenvolver sem servidor, resolvi me cadastrar na Amazon, ganhei uns créditos e fui testando e então fiquei, porque gostei dos resultados". [P5] 
"Entrei na cloud por curiosidade e facilidade, tive um problema como minha IDE e então resolvi jogar meu código para nuvem e gostei a facilidade e comodidade que o desenvolvedor tem. [P4]

A Tabela 5 resume como os aspectos individuais, todos positivos, que influenciam na adoção de um ecossistema na nuvem.

\begin{tabular}{|l|l|l|}
\hline Positivo & Negativo & Ambíguos \\
\hline Aprendizagem / Curiosidade (P4) & & \\
\hline Desafio pessoal (P13, P5) & & \\
\hline Reconhecimento (P13, P4) & & \\
\hline
\end{tabular}

Tabela 5. Principais aspectos individuais.

\section{Discussão}

A Tabela 6 apresenta uma visão geral de todos os aspectos identificados na seção anterior indicando se os mesmos são positivos, negativos ou ambíguos e também se cada aspecto é um aspecto técnico, social, de negócios ou individual. Analisando esta tabela, percebese que os aspectos individuais foram todos positivos. Isto, de certa forma, é esperado pois os entrevistados todos decidiram adotar plataformas em nuvem. Caso desenvolvedores que não tivessem adotado estas plataformas, é possível que aspectos negativos sobre isto fossem identificados. É importante observar também que nenhum fator foi reportado unicamente como negativo, isto é, os fatores identificados podem ser identificados como negativos e positivos dependendo do entrevistado.

\begin{tabular}{|l|l|l|}
\hline Tipo de Aspecto & Positivo & Ambíguos \\
\hline Aspectos Técnicos & Boas APIs & Documentação \\
\cline { 2 - 3 } & Programação sem Servidor & $\begin{array}{l}\text { Infraestrutura de } \\
\text { Desenvolvimento }\end{array}$ \\
\hline Aspectos Sociais & Evangelização da plataforma & Engajamento da comunidade \\
\hline Aspectos Individuais & Aprendizagem & \\
\cline { 2 - 3 } & Desafio pessoal & \\
\cline { 2 - 3 } & Reconhecimento & \\
\hline Aspectos de Negócios & Mercado de trabalho & Precificação \\
\cline { 2 - 3 } & & Rentabilidade \\
\hline
\end{tabular}

Tabela 6. Disposição dos fatores positivos e negativos de acordo com os aspectos

Existem poucos trabalhos na literatura relacionados aos fatores de adoção dos ecossistemas de softwares. Miranda (2016) realizou um estudo qualitativo que buscou analisar e entender as motivações e critérios que possam influenciar engenheiros de software a adotar um determinado ecossistemas de software móvel. Os resultados encontrados na presente pesquisa se assemelham em alguns pontos com os resultados do estudo de Miranda (2016), com destaque para os fatores sociais, que incluem a comunidade de desenvolvedores. 
Ferreira (2016) também estudou ecossistemas de software móveis, porém utilizando como framework conceitual a teoria de difusão da inovação de Rogers (2003). Os resultados são similares ao de Muller (2016), e consequentemente, similares aos apresentados neste trabalho. Um fator que diferencia este trabalho das pesquisas do Ferreira (2016) e Miranda (2016) foi a descoberta de um aspecto não mencionado nas pesquisas citadas: o aspecto individual que revelou-se importante durante o estudo. Outro ponto que merece destaque é o aspecto de negócios: alguns entrevistados citaram que a mudança de mercado influenciou na tomada de decisão e eles perceberam que existem muitas vagas de emprego disponíveis para desenvolvedores na nuvem.

Esta pesquisa identificou os seguintes aspectos: o desafio de aprender coisas novas, a identificação e comprometimento com a comunidade, a diversão durante o processo de desenvolvimento e a influência de terceiros, como amigos, colegas de graduação ou de trabalho. Esses aspectos foram igualmente relatados em outros estudos, como o de Steglich (2019), Miranda (2016) e Ferreira (2016). Outros aspectos incluem a comunidade, seja ela online ou local, além do tamanho e qualidade da comunidade de desenvolvedores. Por fim, foi também identificada a precificação da plataforma, aspecto não identificado nos estudos anteriores.

Koch and Kerschbaum (2014) relatam que as influências entre as plataformas Android e iOS são diferentes. Como exemplo, pode-se citar os entrevistados que desenvolvem para Android, os quais relataram que a remuneração é um fator não relevante, diferente dos desenvolvedores iOS que mencionam a remuneração como fator importante para adotar um ecossistema móvel. Neste estudo, a remuneração não apareceu diretamente como um fator de influência. Entretanto, a possibilidade de conseguir um emprego como desenvolvedor na nuvem mostrou-se relevante para os entrevistados.

De um modo geral, esta pesquisa analisa e relata diferentes fatores que influenciam na adoção e permanência em um ecossistema de software na nuvem. Estes fatores, como dito anteriormente, de alguma forma se relacionam e contribuem um com o outro. Além disso, é importante ressltar que um fator isolado não tem tanto impacto para iniciar e desistir de desenvolver para uma plataforma. De uma maneira geral, conjuntos de fatores influenciam as pessoas a adotarem um ecossistema em nuvem.

\section{Conclusões}

Os ecossistemas de software estão transformando a forma como software é desenvolvido. O modelo de desenvolvimento em que um produto e serviço é criado dentro de uma única empresa está sendo substituído por um modelo compartilhado, composto por diversas empresas, desenvolvedores internos e externos que colaboram entre si para entregar valor aos seus usuários, formando, assim, um ecossistema em torno das plataformas. Estudos anteriores sobre a adoção de ecossistemas de software focaram no desenvolvimento móvel (Koch e Kerschbaum, 2014) (Miranda, 2016) (Ferreira (2016). Assim, faz-se necessário um estudo focado em ecossistemas de software na nuvem. Este foi o objetivo deste artigo que revelou o importante e complexo papel dos aspectos técnicos, negócio, social e individual na adoção de ecossistemas na nuvem.

\section{Agradecimentos.}

Os autores gostariam de agradecer ao apoio financeiro do $\mathrm{CNPq}$ através do processo [400920/2019-0]. 


\section{Referências}

Computerworld, https://computerworld.com.br/2019/02/04/aws-segue-como-segmentode-crescimento-mais-acelerado-na-amazon/. Acessado em 13/04/2019.

C. Steglich et al., "Social Aspects and How They Influence MSECO Developers," 2019 IEEE/ACM 12th International CHASE Workshop, Montreal, QC, Canada, 2019, pp. 99-106.

Barbosa, O. et al. (2013) Software Ecosystems: Analyzing and Managing Business, Chapter: "A Systematic Mapping Study on Software Ecosystems through a Threedimensional Perspective”. Edward Elgar.

Berk, I., et al. (2010) "Software Ecosystems: A Software Ecosystem Strategy Assessment Model". In: Proceedings of the 4th ECSA, 2nd International Workshop , Copenhagen, pp. 135-142.

Bosch, J. (2009) "From Software Product Lines to Software Ecosystems". In: Proc. of the 13th Intl. Software Product Line Conf. San Francisco, USA, ACM pp. 111-119.

Y. Demchenko (2014) "Federated Access Control in Heterogeneous In- tercloud Environment: Basic Models and Architecture Patterns”. In: Proceedings of IC2E 2014.

Robert, P. Mahowald (2017) IDC Future Scape: "Worldwide Cloud 2017 Predictions. study. International Data Corporation”, 2017.

ROGERS, Everret M. Diffusion of Innovations. 5a. Ed. New York: Free Press, 2003.

Slawik, M. (2018) "Managing Cloud Ecosystems. zur Erlangung des akademischen Grades Doktor der Ingenieurwissenschaften" - Dr.-Ing. - von der Universität Berlin, 2018.

P. T. Krishnan Jose L. (2015) "Ugia Gonzalez. Building Your Next Big Thing with Google Cloud Platform" - 2015. p. 3-12.

Corbin, J., Strauss, A. (2008) "Basics of Qualitative Research: Techniques and Procedures for Developing Grounded Theory" (3rd ed.). Thousand Oaks, CA: Sage

Souza, C., et al. (2016) "The Social Side of Software Platform Ecosystems In" Proceedings of the 2016 CHI Conference Pages 3204-3214. 